\title{
Turbulent Transport Reduction by Zonal Flows: Massively Parallel Simulations
}

\author{
Z. Lin, T. S. Hahm, W. W. Lee, W. M. Tang, and R. B. White \\ Princeton Plasma Physics Laboratory, Princeton University, P. O. Box 451, Princeton, NJ 08543
}

$(5 / 18 / 1998)$

\begin{abstract}
The dynamics of turbulence-driven $\mathbf{E} \times \mathbf{B}$ zonal flows has been systematically studied in fully 3-dimensional gyrokinetic simulations of microturbulence in magnetically-confined toroidal plasmas using recently available massively parallel computers. Linear flow damping simulations exhibit an asymptotic residual flow in agreement with recent analytic calculations. Nonlinear global simulations of instabilities driven by temperature gradients in the ion component of the plasma provide key first principles results supporting the physics picture that turbulence-driven fluctuating $\mathbf{E} \times \mathbf{B}$ zonal flows can significantly reduce turbulent transport.
\end{abstract}

Turbulence shear suppression by $\mathbf{E} \times \mathbf{B}$ flows is the most likely mechanism responsible for the transition to various forms of enhanced confinement regimes observed in magnetically-confined plasmas [1]. Understanding the physical mechanisms of turbulence suppression processes [2,3] and developing techniques to possibly control the turbulence are of interest for many areas of research and particularly for magnetic fusion. Recent experimental data from tokamaks such as TFTR [4] and DIIID [5] show evidence of small radial scale $\mathbf{E} \times \mathbf{B}$ flows which cannot be explained by the existing neoclassical (Coulomb collisional) theory. These observations point to the possibility of a spontaneous generation of $\mathbf{E} \times \mathbf{B}$ zonal flows which in turn regulate the turbulence. The key issue here is what controls the generation of the flows and how strongly the flows affects the turbulent transport which is believed to arise from electrostatic pressuregradient driven instabilities. These highly complex nonlinear phenomena can be most effectively investigated by numerical experiments. One of the most promising approaches is gyrokinetic particle-in-cell simulation [6] which suppress the rapid gyromotion of a charged particle about the magnetic field line. By making use of recent advances of new low-noise numerical algorithms and by taking advantage of the exciting opportunities offered by high-end massively parallel computing power, it has been able to reproduce key features of turbulent transport observed at the core of tokamak plasmas.

The present numerical experiments clearly demonstrate that turbulence-driven fluctuating $\mathbf{E} \times \mathbf{B}$ zonal flows play a crucial role in regulating nonlinear saturation and transport levels. This is in agreement with previous toroidal gyrokinetic and gyrofluid (a fluid model with kinetic effect) simulations of instabilities driven by iontemperature-gradient (ITG) in a local geometry which follows a magnetic field line [7-9]. However, previous global gyrokinetic simulations, which treat the whole plasma volume, either did not include [10] or did not observe [11,12] significant effects of these self-generated flows. Since local simulations are restricted to a fluxtube domain with radially periodic boundary conditions and since they rely on the assumption of scale separation between the turbulence and equilibrium profiles, the key issues of transport scaling and effects of steep pressure profiles in transport barriers can only be effectively studied in global simulations. In this report, nonlinear simulation results from a newly developed global gyrokinetic code [13] yield the important conclusion that turbulencedriven fluctuating $\mathbf{E} \times \mathbf{B}$ flows can significantly reduce the anomalous transport. In order to understand this key process, the dynamics of $\mathbf{E} \times \mathbf{B}$ flows have been systematically analyzed. Linear flow damping simulations exhibit a time asymptotic residual flow in agreement with a recent analytic calculation [14]. The present nonlinear global simulations reveal up to an order of magnitude reduction in ion heat conductivity when the self-generated flows are included. These simulations indicate that the $\mathbf{E} \times \mathbf{B}$ flows contain significant components with radial scales and frequencies comparable to those of the turbulence. Insights from these studies serve to resolve the outstanding differences in the flow dynamics observed in the global and local simulations.

A fully three-dimensional global gyrokinetic toroidal code (GTC) [13] based on the low noise nonlinear $\delta f$ scheme [15] for studying both turbulence and neoclassical physics has been developed. The implementation of a general geometry Poisson solver [16] and Hamiltonian guiding center equations of motion [17] in magnetic coordinates [18] have enabled the code to efficiently treat both advanced axisymmetric and nonaxisymmetric configurations using realistic numerical MHD equilibria. This global code takes into account equilibrium profile variation effects and has superior particle noise properties. Furthermore, the unique capability of a single code to simulate both a full poloidal cross section and an annular box with radially periodic boundary conditions pro- 
vides a connection between global and local simulations.

The GTC has been implemented as a platformindependent program and has achieved nearly perfect scalability on various massively parallel processing (MPP) systems (e.g., CRAY-T3E and Origin-2000 supercomputers) as demonstrated in Fig. 1. This scalability enables the code to fully utilize the rapidly increasing MPP computer power which presently allows routine nonlinear simulations of more than $10^{8}$ particles with realistic plasmas parameters of existing fusion experiments to address important physics issues previously inaccessible. Nevertheless, more than two orders of magnitude increase in computing power will be required to assess turbulent transport properties of reactor-relevant plasmas with additional key features such as nonadiabatic electron response, electromagnetic perturbations, and larger system size. These demanding computational capabilities will be delivered by next generation MPP systems with thousands of computing elements whose power can only be effectively utilized by a scalable program such as the GTC.

The GTC code has been rigorously benchmarked against earlier analytic and computational models for neoclassical transport [19] and toroidal ITG simulations. Linear ITG growth rates and real frequencies are found to agree well with results from linear gyrofluid code [7] calculations, and steady state transport results are consistent with those from global gyrokinetic Cartesian code [10] nonlinear simulations when turbulence-driven $\mathbf{E} \times \mathbf{B}$ flows are suppressed. Extensive convergence studies with respect to the size of each time step, the number of grid points, and the number of particles in nonlinear simulations using representative parameters from tokamak experiments have been carried out. In particular, the convergence of the ion heat conductivity $\chi_{i}$ and fluctuation energy level with respect to the number of particles has been demonstrated in nonlinear simulations using a total of 20 million grid points in the $(\psi, \theta, \zeta)$ space with $\psi, \theta$, and $\zeta$ being the toroidal flux function and poloidal and toroidal angles. The fact that the ion heat conductivity remains unchanged when the number of particles is increased from 32 million to 80 million supports the conclusion that $\chi_{i}$ is well converged. Similar convergence of the fluctuation energy is obtained with 80 million particles.

With rigorous validity checks completed, the GTC was next applied to the analysis of turbulence-generated zonal flows in toroidal plasmas. These flows are driven by the flux-surface-averaged radially local charge separation, and mainly in the poloidal direction for high aspect ratio devices. Recently, Rosenbluth and Hinton [14] have pointed out the importance of an accurate prediction of the undamped component of poloidal flows in determining the transport level in nonlinear turbulence simulations. In that work, an illuminating test problem for predicting the residual flow level in response to an initial flow perturbation introduced by a flux-surface-averaged charge separation was solved analytically [14]. Here it is tested in gyrokinetic particle simulations by solving the toroidal gyrokinetic equation [20] with an initial source which is constant on a flux surface and introduces a perturbation of the poloidal flow. This flow is relaxed through the transit time magnetic pumping effect [21] followed by a slower damped oscillation with a characteristic frequency corresponding to that of the geodesic acoustic mode (GAM) [22]. The residual level of this flow measured from the simulation agrees well with the theoretical prediction [14]. These are the first global gyrokinetic code results which provide the observation of the GAM oscillation and a rigorous benchmark of the residual flow level. The studies further support the accuracy of the GTC in treating the dynamics of $\mathbf{E} \times \mathbf{B}$ flows.

Turbulence-driven zonal flows are now self-consistently included in the nonlinear simulations of toroidal ITG instabilities. The flows are generated by the Reynolds stress [23] and can be considered as a nonlinear instability associated with inverse cascade of the turbulent spectra [24]. The present global simulations produce fluctuating $\mathbf{E} \times \mathbf{B}$ flows containing significant components with radial scales and frequencies comparable to those of the turbulence. This is in qualitative agreement with flux-tube simulations [7,8], and clearly demonstrates the possible existence and the importance of such fluctuating flows, which cannot be explained by the existing neoclassical theory. These simulations use representative parameters of DIII-D $H$-mode core plasmas which have a peak ion temperature gradient at $r=0.5 a$ with the following local parameters: $R_{0} / L_{T}=6.9, \eta_{i} \equiv L_{n} / L_{T}=3.2$, $q=1.4, \hat{s} \equiv(r / q)(d q / d r)=0.78, T_{e} / T_{i}=1$, and $\epsilon \equiv a / R_{0}=0.36$. Here $R_{0}$ is the major radius, $L_{T}$ and $L_{n}$ are the temperature and density gradient scale lengths, respectively, $T_{i}$ is the ion temperature, and $T_{e}$ is the electron temperature. The size of the plasma column is $a=160 \rho_{i}$ where $\rho_{i}$ is the thermal ion gyroradius measured at $r=0.5 a$. ITG modes are unstable with these parameters. The simplified physics model includes: a parabolic $q$ profile, a circular cross section, no impurities, and an electron response with $\delta n_{e} / n_{0}=e(\Phi-<\Phi>) / T_{e}$, where $<\cdots>$ represents a flux surface average. A typical nonlinear simulation calculates 5000 time steps of the trajectories of 100 million guiding centers interacting with the self-consistent turbulent field which is discretized by 25 million grid points in a 3-dimensional configuration. The instabilities evolve from a linear phase of growth to a quasilinear saturation with a peak transport level, and finally to a fully developed turbulence with a steady state transport level which is insensitive to initial conditions (Fig. 2). To illustrate the effects of these flows on transport, simulations of the same set of parameters are also carried out with $\mathbf{E} \times \mathbf{B}$ flows suppressed by forcing $\langle\Phi\rangle=0$. The time history 
of $\chi_{i}$ from the simulation with turbulence-driven $\mathbf{E} \times \mathbf{B}$ flows included are compared in Fig. 2 to that from the simulation with the flows suppressed. It is found that a significant reduction (up to an order of magnitude) of steady state ion heat conductivity occurs when $\mathbf{E} \times \mathbf{B}$ flows are retained in global gyrokinetic simulations. This important effect either has not been included [10] or has been reported to be weak $[11,12]$ in steady state transport from previous global gyrokinetic simulations.

A key mechanism of reducing transport by $\mathbf{E} \times \mathbf{B}$ flows is the breaking of turbulent eddies, and consequently, the reduction of the radial decorrelation length [2]. This process is visualized in Fig. 3 which compares the poloidal contour plots of the fluctuation potential in the nonlinear phase from the simulation carried out with $\mathbf{E} \times \mathbf{B}$ flows included to the one with the flows suppressed. The dominant $k_{\theta}=0$ mode is filtered out in these contour plots to highlight the differences in the turbulent eddy size. In both cases, the amplitude of fluctuations is highest at larger major radius where the drive of instabilities is strongest. Similar structures are observed in the linear phase for both cases. $\mathbf{E} \times \mathbf{B}$ flows, which are linearly stable, are generated in the nonlinear saturation stage and begin to tear apart the turbulent eddies. In steady state, the fluctuations are observed to be locally isotropic in radial and poloidal directions when $\mathbf{E} \times \mathbf{B}$ flows are included in the simulations, while the turbulent eddies are elongated along the radial direction when the flows are suppressed (Fig. 3). The fact that the breaking of turbulent eddies by $\mathbf{E} \times \mathbf{B}$ flows results predominantly in the reduction of the radial correlation length is also reflected in the observed flow-induced broadening of the fluctuation spectrum of $k_{r}$. These trends are in qualitative agreement with theoretical predictions $[2,3]$. The reduction of $\chi_{i}$ also results from the reduced fluctuation levels due to the turbulence suppression caused by the selfgenerated flows. Finally, the $\mathbf{E} \times \mathbf{B}$ flows also broaden the oscillation frequency of the individual mode which would otherwise possesses a coherent mode history corresponding to a frequency spectrum with a well-defined peak $[13]$.

Fluctuating flows with a radial characteristic scale length comparable to that of the ambient turbulence have been generated in flux-tube simulations [7,8]. On the other hand, $\mathbf{E} \times \mathbf{B}$ flows with scale lengths of the system size have been the dominant feature in previous global gyrokinetic simulations $[11,12]$ although the finer scale flows began to appear in a larger system size [12]. In addition, global codes typically yield a much lower value of $\chi_{i}$ than that from flux-tube codes. These fundamentally different trends have been attributed to differences between global and local simulation models. Specifically, the whole plasma volume is simulated in global codes with pressure gradient profile variation and fixed boundary conditions, while local codes have a simulation domain which covers a few turbulent decorrelation lengths with a uniform pressure gradient and utilize radially periodic boundary conditions. In the present studies, simulations using both global and annular geometry with a variety of boundary conditions have been carried out to address these differences. The perturbed electrostatic potential is set to zero at the boundary in all global simulations and a radially periodic boundary condition similar to that of the flux-tube simulations $[7,8]$ is implemented in the annulus simulations. The profile of the pressure gradient is varied in the global simulations to distinguish the effects of profile variations from that of boundary conditions. When the profile of the pressure gradient is broad in global simulations, the fine scale components of $\mathbf{E} \times \mathbf{B}$ flows with radial characteristic scale length comparable to the turbulence decorrelation length become dominant. Similar structures of the $\mathbf{E} \times \mathbf{B}$ shearing parameter $\omega_{E} \equiv d v_{E \times B} / d r$ are observed from both the present global and local simulations as shown in Fig. 4. Furthermore, good agreement in the ion heat conductivities are obtained between the local and global simulations with a broad pressure gradient profile. These results indicate that the radially periodic boundary conditions in local codes are not responsible for the differences between the trends observed in local and global simulations-at least for the cases studied. As the variation in the pressure gradient becomes stronger in the global simulation, a static single-well structure in $E_{r}$, similar to those observed in previous global codes [11,12], emerges to be dominant. At the same time, the ion heat conductivities decrease due to the profile variation effects. This leads to the conclusion that the narrow pressure gradient profile in global codes could be responsible for the differences with the local code results.

In future studies, the dynamics of turbulence-driven zonal flows will be further explored including additional effects associated with collisional damping, nonadiabatic electron response, and electromagnetic perturbations. Realistic simulations will continue to lead to scientific discoveries made possible by the dramatic increase in next generation high performance computing power.

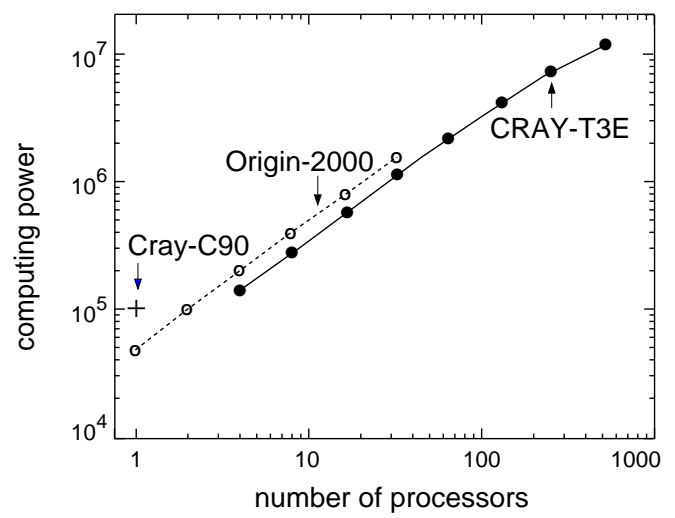


FIG. 1. Performance of the GTC on massively parallel computers (CRAY-T3E and Origin-2000) and vector supercomputer (CRAY-C90). Y-axis is the number of particles which move one step in one second.

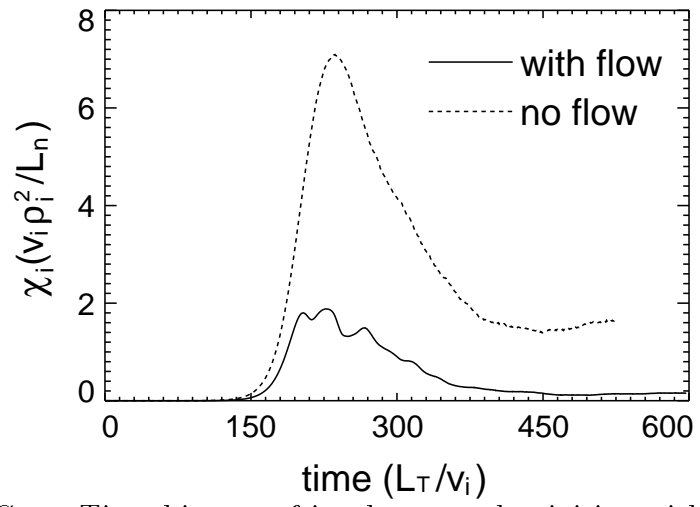

FIG. 2. Time history of ion heat conductivities with and without $\mathbf{E} \times \mathbf{B}$ flows in global simulations.

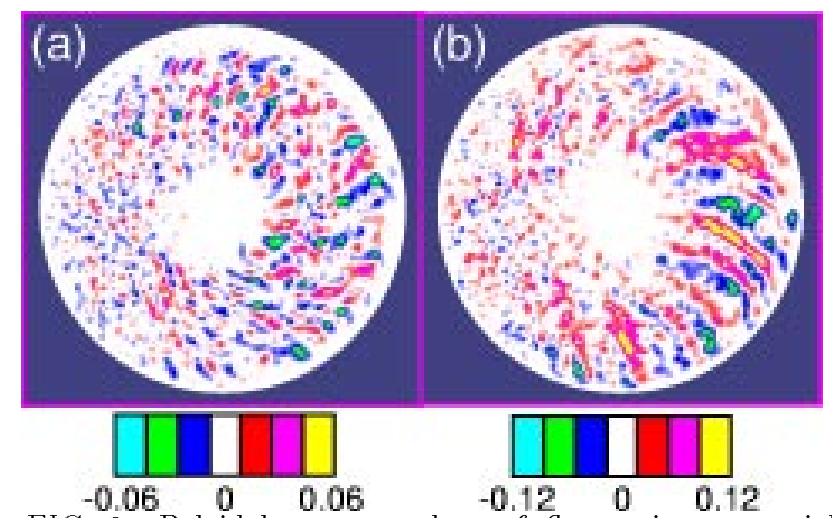

FIG. 3. Poloidal contour plots of fluctuation potential $\left(e \Phi / T_{i}\right)$ in the steady state of nonlinear global simulation with $\mathbf{E} \times \mathbf{B}$ flows included (a) and with the flows suppressed (b).

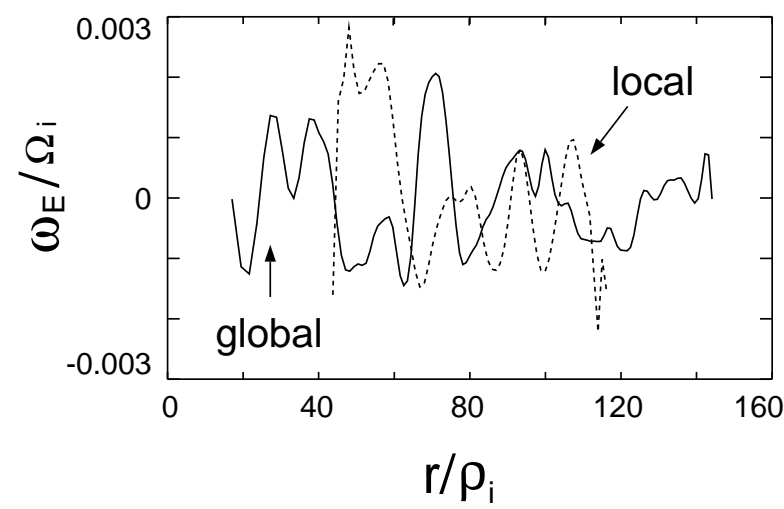

FIG. 4. Instantaneous radial profile of $\mathbf{E} \times \mathbf{B}$ shear in local (dotted) and global (solid) simulations with broad profile.
[1] K. H. Burrell, Phys. Plasmas 4, 1499 (1997); E. Mazzucato et al., Phys. Rev. Lett. 77, 3145 (1996); E. J. Synakowski et al., Phys. Rev. Lett. 78, 2972 (1997); H. Shirai et al., Phys. Plasmas 5, 1712 (1998); G. Wang et al., Phys. Plasmas 5, 1328 (1998).

[2] H. Biglari, P. H. Diamond, and P. W. Terry, Phys. Fluids B 2, 1 (1990).

[3] T. S. Hahm and K. H. Burrell, Phys. Plasmas 2, 1648 (1995).

[4] R. E. Bell, F. M. Levinton, S. H. Batha, E. J. Synakowski, and M. C. Zarnstorff, Plasma Phys. Control. Fusion 40, 609 (1998).

[5] R. J. Groebner, Phys. Fluids B 5, 2343 (1993).

[6] W. W. Lee, Phys. Fluids 26, 556 (1983).

[7] M. A. Beer, PhD dissertation, Princeton University, 1995.

[8] A. M. Dimits, T. J. Williams, J. A. Byers, and B. I. Cohen, Phys. Rev. Lett. 77, 71 (1996).

[9] R. E. Waltz, R. L. Dewar, and X. Garbet, Phys. Plasmas 5, 1784 (1998).

[10] S. E. Parker, W. W. Lee, and R. A. Santoro, Phys. Rev. Lett. 71, 2042 (1993).

[11] J. C. Cummings, PhD dissertation, Princeton University, 1995.

[12] R. D. Sydora, V. K. Decyk, and J. M. Dawson, Plasma Phys. Control. Fusion 38, A281 (1996).

[13] Z. Lin, T. S. Hahm, W. W. Lee, W. M. Tang, and R. B. White, to be submitted to Phys. Plasmas (1998).

[14] M. N. Rosenbluth and F. L. Hinton, Phys. Rev. Lett. 80, 724 (1998).

[15] A. M. Dimits and W. W. Lee, J. Comput. Phys. 107, 309 (1993); S. E. Parker and W. W. Lee, Phys. Fluids B 5, 77 (1993); G. Hu and J. A. Krommes, Phys. Plasmas 1, 863 (1994).

[16] Z. Lin and W. W. Lee, Phys. Rev. E 52, 5646 (1995).

[17] R. B. White and M. S. Chance, Phys. Fluids 27, 2455 (1984).

[18] A. H. Boozer, Phys. Fluids 24, 1999 (1981).

[19] Z. Lin, W. M. Tang, and W. W. Lee, Phys. Rev. Lett. 78, 456 (1997).

[20] T. S. Hahm, Phys. Fluids 31, 2670 (1988); E. A. Freeman and L. Chen, Phys. Fluids 25, 502 (1982).

[21] T. H. Stix, Phys. Fluids 16, 1260 (1973).

[22] N. Winsor, J. L. Johnson, and J. M. Dawson, Phys. Fluids 11, 2448 (1968).

[23] P. H. Diamond and Y. B. Kim, Phys. Fluids B 3, 1626 (1991).

[24] A. Hasegawa and M. Wakatani, Phys. Rev. Lett. 59, 1581 (1987).

[25] This work was supported by DoE Contract No. DEAC02-76CH03073, and in part by an appointment to the U.S. Department of Energy Fusion Energy Postdoctoral Research Program administered by the Oak Ridge Institute for Science and Education. 